\title{
Improved oral bioavailability and gastro-intestinal stability of amphotericin B through fatty acid conjugation approach
}

\author{
Kaushik Thanki†, Tushar Date†, Sanyog Jain* \\ Centre for Pharmaceutical Nanotechnology, Department of Pharmaceutics, National Institute of Pharmaceutical Education \\ and Research (NIPER), Sector 67, SAS Nagar (Mohali), Punjab - 160062, India
}

\section{$\underline{\text { Supporting Information }}$}

\section{Hydrolysis in liver homogenate}

The hydrolytic cleavage of AmB-OA in presence of liver homogenates revealed formation of parent compound along with other intermediate products. The mass spectrum of representative sample confirming peaks at $1295\left(\mathrm{M}+\mathrm{Na}^{+}\right.$peak of AmB-OA), $1191\left(\mathrm{M}+\mathrm{H}_{2} \mathrm{O}\right.$ peak of BOC cleaved conjugate), 1046 (cleavage of oleyl group), $963\left(\mathrm{M}+\mathrm{K}^{+}\right.$peak of AmB), 760 (cleavage of mycosamine moiety from AmB), 798 (hydrolysis followed by oxidation of secondary alcohols of AmB) (Figure S1).

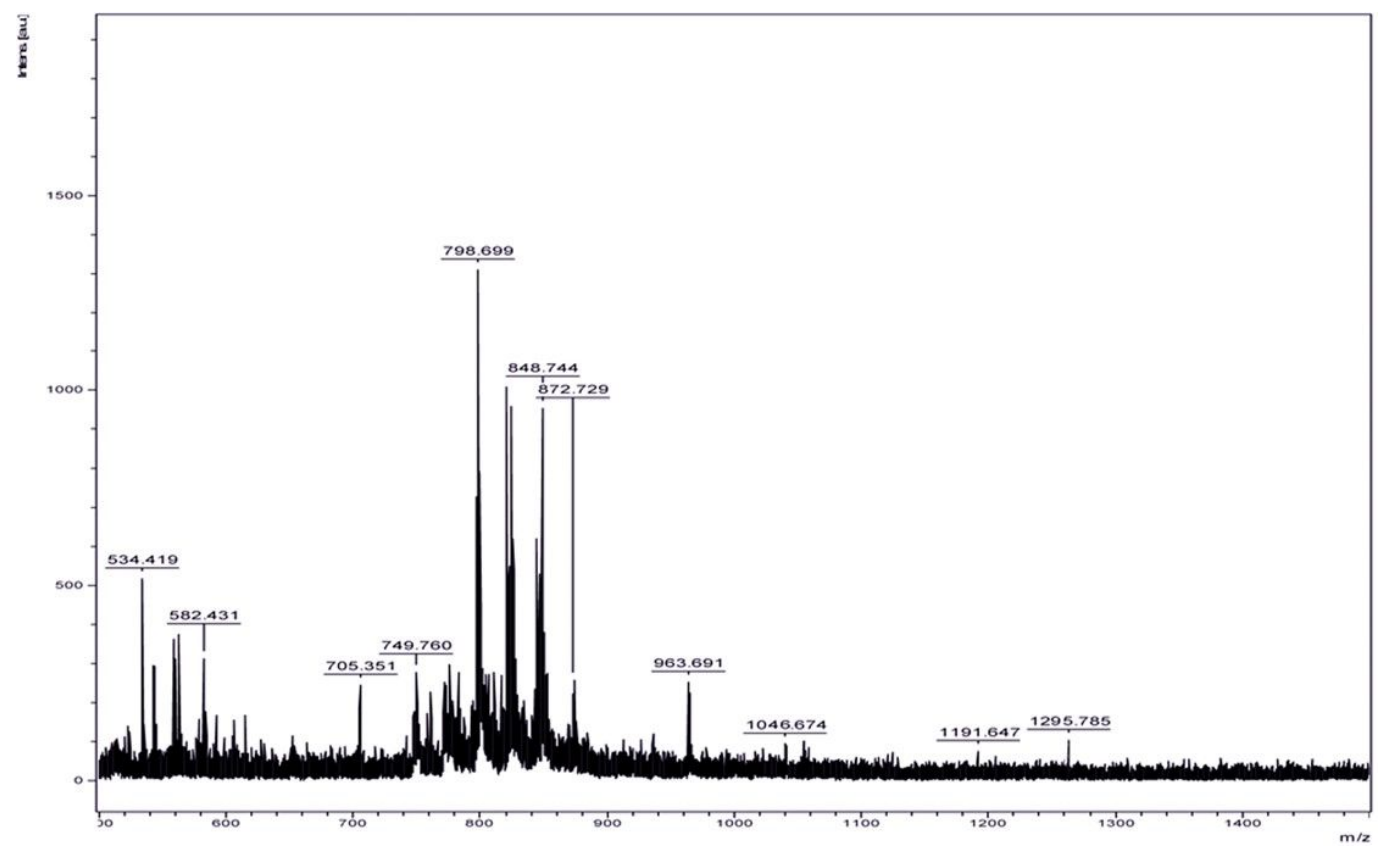

Figure S1: Mass spectrum of AmB-OA sample subjected to hydrolysis studies in presence of liver homogenate 